\title{
High efficiency carbon nanotube thread antennas
}

\author{
E. Amram Bengio, ${ }^{1}$ Damir Senic, ${ }^{2}$ Lauren W. Taylor, ${ }^{1}$ Dmitri E. Tsentalovich, ${ }^{1}$ Peiyu Chen, ${ }^{3}$ \\ Christopher L. Holloway, ${ }^{2}$ Aydin Babakhani, ${ }^{3}$ Christian J. Long, ${ }^{2}$ David R. Novotny, ${ }^{2}$ \\ James C. Booth, ${ }^{2}$ Nathan D. Orloff, ${ }^{2}$ and Matteo Pasquali ${ }^{1, a)}$ \\ ${ }^{1}$ Department of Chemical and Biomolecular Engineering, Rice University, Houston, Texas 77005, USA \\ ${ }^{2}$ National Institute of Standards and Technology, Communications Technology Laboratory, Boulder, \\ Colorado 80305, USA \\ ${ }^{3}$ Department of Electrical and Computer Engineering, Rice University, Houston, Texas 77005, USA
}

(Received 23 June 2017; accepted 24 September 2017; published online 20 October 2017)

\begin{abstract}
Although previous research has explored the underlying theory of high-frequency behavior of carbon nanotubes (CNTs) and CNT bundles for antennas, there is a gap in the literature for direct experimental measurements of radiation efficiency. These measurements are crucial for any practical application of CNT materials in wireless communication. In this letter, we report a measurement technique to accurately characterize the radiation efficiency of $\lambda / 4$ monopole antennas made from the CNT thread. We measure the highest absolute values of radiation efficiency for CNT antennas of any type, matching that of copper wire. To capture the weight savings, we propose a specific radiation efficiency metric and show that these CNT antennas exceed copper's performance by over an order of magnitude at $1 \mathrm{GHz}$ and $2.4 \mathrm{GHz}$. We also report direct experimental observation that, contrary to metals, the radiation efficiency of the CNT thread improves significantly at higher frequencies. These results pave the way for practical applications of CNT thread antennas, particularly in the aerospace and wearable electronics industries where weight saving is a priority. Published by AIP Publishing. https://doi.org/10.1063/1.4991822
\end{abstract}

Since their discovery in $1991,{ }^{1}$ it has been a challenge to obtain the unique mechanical, ${ }^{2}$ thermal, ${ }^{3}$ and electrical ${ }^{4}$ single molecule properties of carbon nanotubes (CNTs) at the macro-scale. Due to this unique combination of properties, CNT materials have long been considered for their potential as mechanically durable, lightweight RF antennas. Despite a fascinating study in which an individual CNT was used simultaneously as an antenna, a tunable band-pass filter, an amplifier, and a demodulator in a functional radio, ${ }^{5}$ physical measurements on individual CNTs have shown poor performance as microwave antennas due to their high quantum capacitance and input impedance. ${ }^{6}$ Nevertheless, experimental studies on the high-frequency electrical properties of CNT bundles have shown that their inductance scales inversely with the number of individual CNTs within the bundle. $^{7}$ Because of these results, efforts are underway to use macro-scale CNT materials such as CNT films and composites for RF patch antennas, ${ }^{8-12}$ however, device-level performance, particularly in the form of a sufficiently high radiation efficiency, is still inadequate or unreported in all published works.

Published studies of CNT thread antennas are sparse and primarily consist of modeling. ${ }^{13-15}$ Keller et al. ${ }^{16}$ deducted from gain measurements that dipole antennas manufactured from dry-spun CNT fibers have low radiation efficiency ( $\sim 26 \%$ of equivalent copper antennas), predicting that even a CNT rope five times thicker than an equivalent copper wire would still only be $\sim 65 \%$ as efficient at $2.45 \mathrm{GHz}$. However, theory predicts that the CNT thread antenna performance should scale with the density and conductivity of the CNT thread; ${ }^{13}$ therefore, highly dense and conductive CNT fibers

\footnotetext{
a) Author to whom correspondence should be addressed: mp@rice.edu
}

may be key to obtaining high radiation efficiencies in CNT thread antennas.

Here, we show that viable CNT thread antennas can be manufactured using densely packed and moderately conducting $(\sim 0.3 \mathrm{MS} / \mathrm{m}) \mathrm{CNT}$ thread. By using a "plug-and-play" test fixture that facilitates reproducible measurements of radiation efficiencies of soft conductors in a reverberation chamber, ${ }^{17}$ we show that, compared to equivalent copper controls, these antennas perform well at communication frequencies ( $1 \mathrm{GHz}$ and $2.4 \mathrm{GHz}$ ) used in wireless medical devices and local area networks (WLAN). At $2.4 \mathrm{GHz}, \mathrm{CNT}$ thread matches the absolute performance of copper wire of the same thickness. We also find that the frequencydependent effective resistance of the CNT thread scales inversely with the square root of frequency, suggesting a relationship with the skin depth, typically observed in bulk metallic conductors.

We fabricated CNT fibers by wet spinning from chlorosulfonic acid, ${ }^{18}$ using CNTs from Meijo, Japan, grade EC 2.0, purified with $\mathrm{H}_{2} \mathrm{O}_{2}$ and $\mathrm{HCl}$ (see supplementary material). ${ }^{19}$ These CNTs have highly graphitic character and high aspect ratio, which is important for attaining high fiber conductivity. ${ }^{20}$ After stabilization, individual fibers were twisted together to form a thread using a simple Boy Scout ropemaking technique. ${ }^{21} \mathrm{We}$ selected the quarter-wavelength monopole configuration for this study because it is the simplest wire antenna to manufacture and is also equivalent to a dipole. For our experiments, we studied how the number of CNT fibers $(\mathrm{N}=8,16,24,32,64$, and 128) affected the CNT thread antenna performance. The twist of the thread ( $\sim 1$ turn $/ \mathrm{mm}$ and $\sim 2$ turns $/ \mathrm{mm}$ for low and high twist, respectively) was also tested and found to have no significant impact on DC resistance or radiation efficiency; hence, we 
TABLE I. Experimental properties of the CNT thread antennas and nominally identical copper antennas. The table contains the type of material used (Sample), the number of filaments (Filaments), the DC resistance of the antenna (R), the cross-sectional area (A), the DC conductivity $(\sigma)$, and the density $(\rho)$.

\begin{tabular}{|c|c|c|c|c|c|}
\hline Sample & Filaments & $\mathrm{R}(\Omega)$ & $\mathrm{A}\left(\mu \mathrm{m}^{2}\right)$ & $\sigma\left(10^{5} \mathrm{~S} \mathrm{~m}^{-1}\right)$ & $\rho\left(\mathrm{g} \mathrm{cm}^{-3}\right)$ \\
\hline Copper & $30 \mathrm{AWG} \mathrm{Cu}$ & 0.03 & 49087 & 568 & 8.9 \\
\hline CNT & 8 & $46.7 \pm 0.5$ & $4930 \pm 60$ & $3.00 \pm 0.05$ & $0.81 \pm 0.08$ \\
\hline CNT & 16 & $27.4 \pm 0.4$ & $9860 \pm 120$ & $2.56 \pm 0.05$ & $0.69 \pm 0.01$ \\
\hline CNT & 24 & $21.4 \pm 1.1$ & $14780 \pm 170$ & $2.18 \pm 0.11$ & $0.82 \pm 0.01$ \\
\hline CNT & 32 & $13.4 \pm 0.1$ & $19710 \pm 220$ & $2.61 \pm 0.04$ & $0.74 \pm 0.02$ \\
\hline CNT & 64 & $6.4 \pm 0.2$ & $39420 \pm 450$ & $2.73 \pm 0.03$ & $0.81 \pm 0.01$ \\
\hline $\mathrm{CNT}$ & 128 & $3.2 \pm 0.1$ & $78850 \pm 900$ & $2.73 \pm 0.03$ & $0.74 \pm 0.02$ \\
\hline
\end{tabular}

averaged over this variable [(a very small but systematic impact was observed on the depth of the $S_{11}$ resonance, see Fig. 2(c)]. After fabrication, we cut (by razor blade) the multifilament thread to $75 \pm 0.5 \mathrm{~mm}$ and $31 \pm 0.5 \mathrm{~mm}$ long segments to achieve the desired antenna resonant frequencies at $1 \mathrm{GHz}$ and $2.4 \mathrm{GHz}$, respectively. We also made copper control antennas from 30 American Wire Gauge (AWG) copper wire (254 $\mu \mathrm{m}$ diameter) with identical lengths (the minimum gauge typically used in copper because of mechanical robustness considerations). Table I reports the dimensions and material parameters of each antenna. As expected, the DC resistance $(R)$ of the CNT thread depends inversely on the cross-sectional area $(A)$, measured via SEM (Figs. S1 and S2, supplementary material). Note that the DC conductivity of the single filament fiber is about an order of magnitude lower than high-conductivity CNT fibers ${ }^{18}$ due to different sources of CNT starting material and un-optimized purification.

Although the CNT thread properties were uniform, we observed that antenna-to-antenna reproducibility depended on the handling of the antenna, due to variability in both the electrical connection between the CNT thread and the SubMiniature version A (SMA) panel connector as well as the flexible nature of the antenna. To overcome these issues, we developed a "plug-n-play" test fixture (Fig. 1), which used the same $30 \times 30 \mathrm{~cm}$ aluminum ground plane and SMA panel connector for all experiments. The test fixture had a female SMA connector attached to the ground plane with silver epoxy. The CNT thread (or copper wire) was attached to a

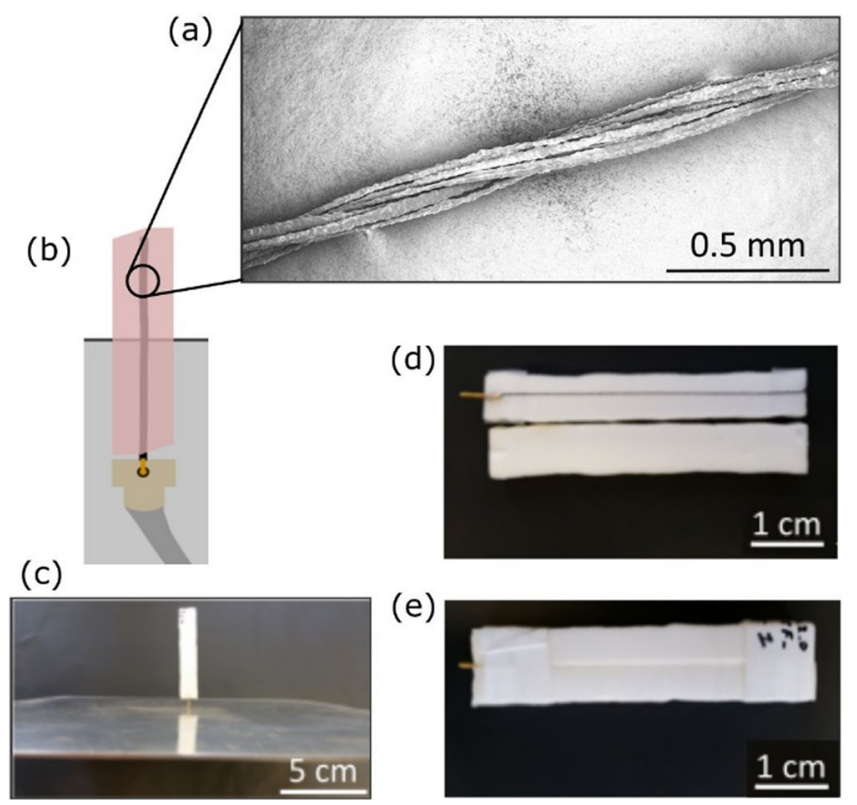

FIG. 1. Experimental configuration for testing CNT thread monopole antennas. (a) SEM micrograph of the 8 filament high-twist CNT thread, (b) schematic representation of the monopole antenna, (c) Rohacell HF/CNT antenna plugged into the $\mathrm{Al}$ ground plane, (d) CNT thread attached to brass pin connector, and (e) Rohacell HF/CNT thread final assembly.

male pin with silver epoxy and then "sandwiched" between two pieces of low loss, low permittivity foam block (Rohacell $31 \mathrm{HF}$ ) with a relative dielectric constant of approximately 1.002. The foam blocks kept the thread (or copper wire) taut during measurement. This reduced the sample-to-sample variability to below $1 \%$.

We verified the reproducibility of the test fixture with 2port Vector Network Analyzer (VNA) measurements of the CNT thread antennas (and copper antenna) inside an anechoic chamber with a reference cone antenna (Fig. 2). The resulting difference in the reflection coefficients $\left(\mathrm{S}_{11}\right)$ between three successive connections of each CNT thread antenna was less than $3 \mathrm{~dB}$, which is small compared to the magnitude of the reflection coefficient $(>12 \mathrm{~dB}$ ). Contrary to a previous report, ${ }^{16}$ we did not observe a systematic frequency downshift, although we did notice small shifts $(\sim 10$ to $100 \mathrm{MHz}$ ) around the target frequency, consistent with the limitations in our cutting precision $(\sim 0.5 \mathrm{~mm})$. In all but one case, the high twist CNT thread displayed a deeper

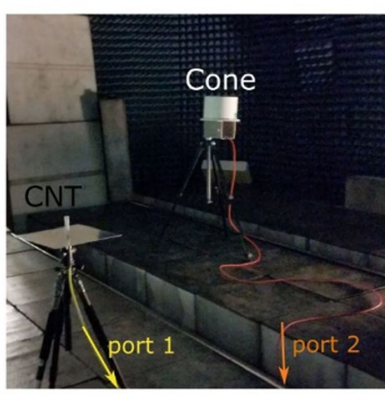

(a)
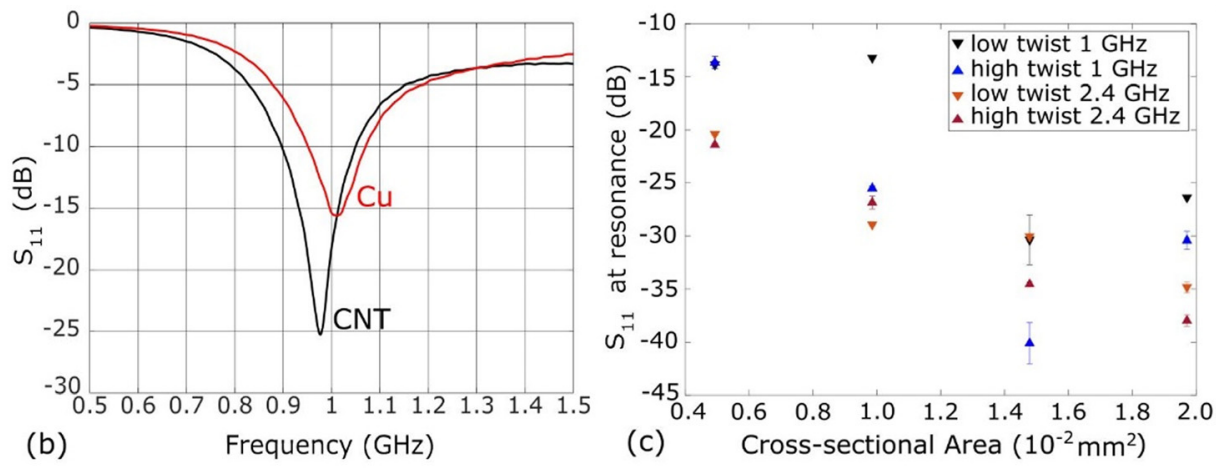

FIG. 2. Validation of the reproducibility of the CNT thread monopole antenna configuration (a) experimental set-up inside the anechoic chamber, (b) representative $S_{11}$ plot of 16 filament low twist CNT thread and 30 AWG Cu wire, and (c) $S_{11}$ values at resonance for all four thinnest CNT thread antennas for three consecutive plugging and un-plugging into the test ground plane. 
resonance than the low twist CNT thread, which could be attributed to better impedance-matching due to reduced capacitance from void spaces. However, this systematic difference [Fig. 2(c)] is minimal since below $-20 \mathrm{~dB}$ less than $1 \%$ of the power fed to the antenna is reflected.

Upon verifying that this set-up gave reproducible results, we performed a series of 2-port VNA measurements inside a reverberation chamber to measure the radiation efficiency of the thread antennas using the two-antenna method. ${ }^{17}$ The CNT thread antenna and a reference horn antenna were placed in a reverberation chamber, which is a high-Q resonant cavity. In this method, a metal paddle rotates to different positions inside the chamber to randomize the electromagnetic standing waves, sampling all possible directional couplings between the two antennas. To accurately measure the radiation efficiency, we averaged the $\mathrm{S}$ parameters over 100 paddle positions. The averaged $S_{11}$ parameters were fitted to a Gaussian curve to obtain a more precise estimate of the resonant frequency (Fig. S3, supplementary material). We verified that the radiation efficiency was independent of the antenna position, which is essential for this technique, and also found that the total efficiency displays the expected maximum at the resonant frequency (Fig. S4, supplementary material). The measurement uncertainties of this type of measurement are discussed in Refs. 17 and 22.

Our measurements show that radiation efficiency increases with the increasing cross-sectional area (Fig. 3), as expected. For an equivalent cross-sectional area of our CNT thread, the radiation efficiency at $2.4 \mathrm{GHz}$ (Wi-Fi) is the same as that of $30 \mathrm{AWG}$ copper wire (within experimental error). This is a rather surprising result, because the DC conductivity of this CNT thread is approximately two orders of magnitude lower than that of copper. At the higher frequency, the gap between the radiation efficiency of copper and that of our CNT thread is narrower. Of course, only a small cross-sectional area of the copper conductor is carrying current, as the skin depth of copper is 2 and $1.3 \mu \mathrm{m}$ at $1 \mathrm{GHz}$ and $2.4 \mathrm{GHz}$, respectively. Unfortunately, little is known about the skin effect in macro-scale CNT materials or if such a mechanism is even significant. CNT fibers may behave like a Litz wire at higher frequencies due to their highly anisotropic conductivity and fibrillar substructure. ${ }^{23}$

Another important observation is that the CNT thread's radiation efficiency improves at the higher frequency of $2.4 \mathrm{GHz}$ compared to its performance at $1 \mathrm{GHz}$. Given that the radiation efficiency does not depend on impedance matching between the antenna and the VNA, ${ }^{17}$ this must be an intrinsic RF property of the antenna. In the absence of a first-principles physical description, we attempt to understand this behavior through a simple model of these $\lambda / 4$ monopole CNT antennas' radiation efficiency, shown in Eq. (1), a modified version of the standard definition of radiation efficiency. $^{24}$

$$
\eta_{r}=\frac{R_{r}}{R_{r}+k R_{l}+R_{x}} .
$$

Here, $R_{r}$ is the antenna's radiation resistance, assumed to be $36.5 \Omega$ as theoretically predicted for a $\lambda / 4$ monopole, ${ }^{24} R_{l}$ is the DC resistance of the CNT thread (listed in Table I), $R_{x}$ is a parameter that encompasses all other resistive losses in the antenna that are not occurring in the CNT thread (i.e., resistive losses in all of the metallic components including the connector, ground plane, and silver epoxy), and $k$ is a dimensionless pre-factor describing the frequency-dependence of the CNT thread resistance. Figure 3 shows that a least-squares fit yields a good RMSE value for the $1 \mathrm{GHz}$ dataset $(2.4 \%)$ and an acceptable RMSE value for the $2.4 \mathrm{GHz}$ dataset (5.2\%). The value of $R_{x}$ decreases by approximately $7 \Omega$ from $8.2 \Omega$ at $1 \mathrm{GHz}$ to $1.3 \Omega$ at $2.4 \mathrm{GHz}$, which is not expected for the resistances of the metallic components. It is possible that this is simply capturing the decrease in losses due to our finite ground plane, since the ground plane size is approximately equal to the wavelength of EM radiation at $1 \mathrm{GHz}$ but is over twice as large as the wavelength at $2.4 \mathrm{GHz}$. Thus, it is possible that at least one component of the measured increase in radiation efficiency of the antennas is not due to an intrinsic property of the CNT material itself. The inverse square root of the ratio between the two fitted $k$ factors is $(0.51 / 0.93)^{0.5}$ $=0.74$, consistent with the inverse square root dependence on frequency $(1 / 2.4)^{0.5}=0.65$, indicating that the skin depth may indeed be responsible for the effect, which would warrant further testing at higher frequencies. Beyond the fundamental interest, if such a trend extends to higher frequencies (35-50 GHz range), then CNT macro-structures could serve as the material of choice for the telecommunication industry's next generation $(5 \mathrm{G})$ antennas.

In summary, CNT thread antennas fabricated from moderately conductive CNT fibers match the radiation efficiency of copper control antennas while saving about an order of magnitude in antenna weight. To capture the tradeoff between weight savings and radiation efficiency, we normalize the radiation efficiency by the weight of the radiating element (copper wire or CNT thread), which we call the specific radiation efficiency (Fig. 4). By this metric, CNT thread antennas are $\sim 20$ times more efficient than copper

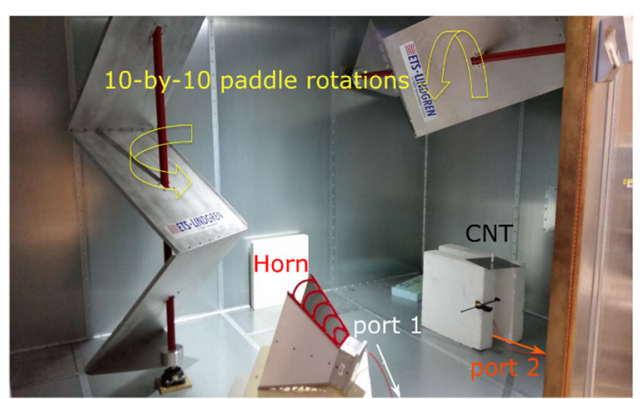

(a)

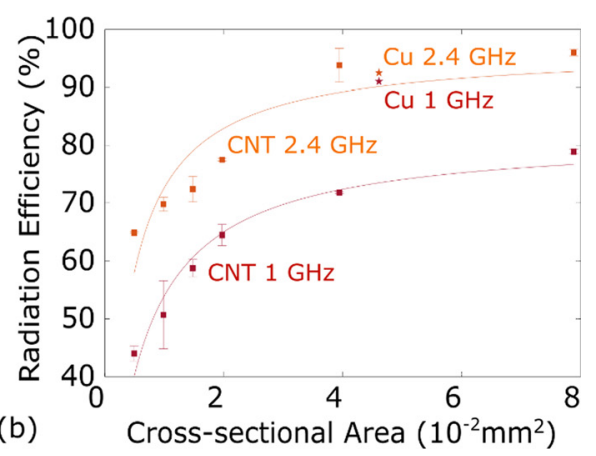

FIG. 3. (a) Measurement set-up in the reverberation chamber. (b) Summary of radiation efficiency measurements on CNT thread (squares) and $\mathrm{Cu}$ control antennas (stars). The error bars were obtained by averaging the high twist and low twist thread efficiencies. 


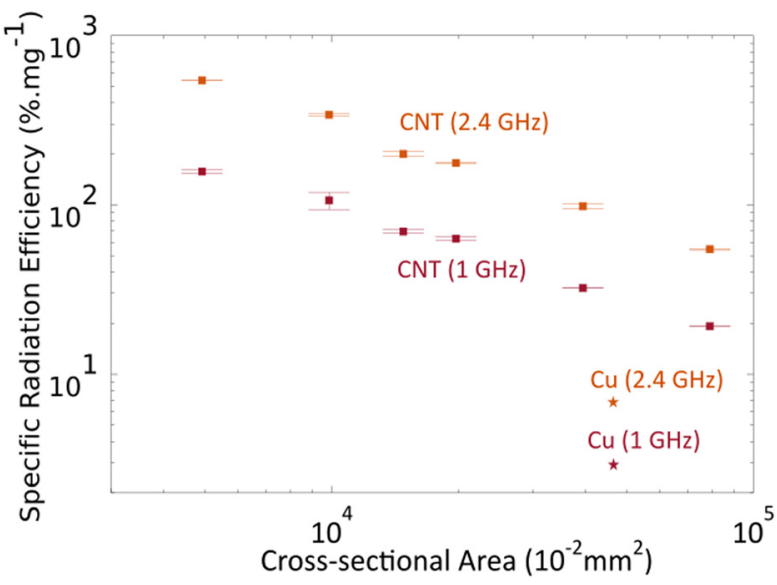

FIG. 4. Plot of specific radiation efficiencies for the CNT thread (squares) and copper antennas (stars).

wire. This demonstrates that at wireless communication frequencies, copper wire antennas can be replaced with CNT thread antennas without impacting performance, while decreasing weight. Although copper wire thinner than 30 AWG could provide identical performance at lower weight at these frequencies, such an antenna would be extremely susceptible to mechanical damage due to copper's high ductility and low tensile strength. While not problematic for stationary antennas, e.g., in ground stations, mechanical robustness is important for antennas in weight-sensitive mobile assemblies, such as aircrafts or satellites. Mechanical robustness and flexibility are even more critical in wearable electronics, where copper's and other metals' poor fatigue behavior poses significant challenges for researchers vying to integrate antennas into clothing.

See supplementary material for details on CNT material purification and fiber-spinning, reverberation chamber and VNA measurements, CNT material characterization, and supplementary figures cited in the manuscript.

This work was supported by U.S. Air Force research Grant No. FA9550-15-1-0370. Lauren W. Taylor would like to acknowledge support under and awarded by DoD, Air Force Office of Scientific Research, National Defense Science and Engineering Graduate (NDSEG) Fellowship, 32 CFR 168a.

The authors declare the following competing financial interest(s): Matteo Pasquali and Dmitri Tsentalovich have a financial interest in DexMat, Inc., which is commercializing solution-spun CNT fibers.

${ }^{1}$ S. Iijima, "Helical microtubules of graphitic carbon," Nature 354, 56-58 (1991).

${ }^{2}$ M.-F. Yu, B. S. Files, S. Arepalli, and R. S. Ruoff, "Tensile loading of ropes of single wall carbon nanotubes and their mechanical properties," Phys. Rev. Lett. 84, 5552 (2000).
${ }^{3}$ S. Berber, Y.-K. Kwon, and D. Tománek, "Unusually high thermal conductivity of carbon nanotubes," Phys. Rev. Lett. 84, 4613 (2000).

${ }^{4}$ T. W. Ebbesen, H. J. Lezec, H. Hiura, J. W. Bennett, H. F. Ghaemi, and T. Thio, "Electrical conductivity of individual carbon nanotubes," Nature 382, 54-56 (1996).

${ }^{5}$ K. Jensen, J. Weldon, H. Garcia, and A. Zettl, "Nanotube radio," Nano Lett. 7, 3508-3511 (2007).

${ }^{6} \mathrm{P}$. J. Burke, S. Li, and Z. Yu, "Quantitative theory of nanowire and nanotube antenna performance," IEEE Trans. Nanotechnol. 5, 314-334 (2006).

${ }^{7}$ J. J. Plombon, K. P. O’Brien, F. Gstrein, V. M. Dubin, and Y. Jiao, "Highfrequency electrical properties of individual and bundled carbon nanotubes," Appl. Phys. Lett. 90, 063106 (2007).

${ }^{8}$ S. D. Keller, A. I. Zaghloul, V. Shanov, M. J. Schulz, D. B. Mast, and N. T. Alvarez, "Radiation performance of polarization selective carbon nanotube sheet patch antennas," IEEE Trans. Antennas Propag. 62, 48-55 (2014).

${ }^{9}$ I. Puchades, J. E. Rossi, C. D. Cress, E. Naglich, and B. J. Landi, "Carbon nanotube thin-film antennas," ACS Appl. Mater. Interfaces 8, 20986-20992 (2016).

${ }^{10}$ Y. Bayram, Y. Zhou, B. S. Shim, S. Xu, J. Zhu, N. A. Kotov, and J. L. Volakis, "E-textile conductors and polymer composites for conformal lightweight antennas," IEEE Trans. Antennas Propag. 58, 2732-2736 (2010).

${ }^{11}$ A. Mehdipour, I. D. Rosca, A.-R. Sebak, C. W. Trueman, and S. V. Hoa, "Carbon nanotube composites for wideband millimeter-wave antenna applications," IEEE Trans. Antennas Propag. 59, 3572-3578 (2011).

${ }^{12}$ T. A. Elwi, H. M. Al-Rizzo, D. G. Rucker, E. Dervishi, Z. Li, and A. S. Birias, "Multi-walled carbon nanotube-based RF antennas," Nanotechnology 21, 045301 (2010).

${ }^{13}$ Y. Huang, W.-Y. Yin, and Q. H. Liu, "Performance prediction of carbon nanotube bundle dipole antennas," IEEE Trans. Nanotechnol. 7, 331-337 (2008).

${ }^{14}$ S. Choi and K. Sarabandi, "Performance assessment of bundled carbon nanotube for antenna applications at terahertz frequencies and higher," IEEE Trans. Antennas Propag. 59, 802-809 (2011).

${ }^{15} \mathrm{G}$. W. Hanson, "Fundamental transmitting properties of carbon nanotube antennas," IEEE Trans. Antennas Propag. 53, 3426-3435 (2005).

${ }^{16}$ S. D. Keller, A. I. Zaghloul, V. Shanov, M. J. Schulz, D. B. Mast, and N. T. Alvarez, "Electromagnetic simulation and measurement of carbon nanotube thread dipole antennas," IEEE Trans. Nanotechnol. 13, 394-403 (2014).

${ }^{17}$ C. L. Holloway, H. A. Shah, R. J. Pirkl, W. F. Young, D. A. Hill, and J. Ladbury, "Reverberation chamber techniques for determining the radiation and total efficiency of antennas," IEEE Trans. Antennas Propag. 60, 1758-1770 (2012).

${ }^{18}$ N. Behabtu, C. C. Young, D. E. Tsentalovich, O. Kleinerman, X. Wang, A. W. K. Ma, E. A. Bengio, R. F. ter Waarbeek, J. J. de Jong, R. E. Hoogerwerf et al., "Strong, light, multifunctional fibers of carbon nanotubes with ultrahigh conductivity," Science 339, 182-186 (2013).

${ }^{19}$ Y. Wang, H. Shan, R. H. Hauge, M. Pasquali, and R. E. Smalley, "A highly selective, one-pot purification method for single-walled carbon nanotubes," J. Phys. Chem. B 111, 1249-1252 (2007).

${ }^{20}$ D. E. Tsentalovich, A. W. K. Ma, J. A. Lee, N. Behabtu, E. A. Bengio, A. Choi, J. Hao, Y. Luo, R. J. Headrick, M. J. Green et al., "Relationship of extensional viscosity and liquid crystalline transition to length distribution in carbon nanotube solutions," Macromolecules 49, 681-689 (2016).

${ }^{21}$ BSA Troop, 228, Ropemaking.

${ }^{22}$ D. Senic, K. A. Remley, C.-H. J. Wang, D. F. Williams, C. L. Holloway, D. C. Ribeiro, and A. T. Kirk, "Estimating and reducing uncertainty in reverberation-chamber characterization at millimeter-wave frequencies," IEEE Trans. Antennas Propag. 64, 3130-3140 (2016).

${ }^{23}$ A. Lekawa-Raus, T. Gizewski, J. Patmore, L. Kurzepa, and K. K. Koziol, "Electrical transport in carbon nanotube fibres," Scr. Mater. 131, 112-118 (2016).

${ }^{24} \mathrm{H}$. Visser, Antenna Theory and Applications (Wiley, 2012). 